\title{
A relação da Síndrome de burnout com a ausência de qualidade de vida no trabalho de enfermagem
}

\author{
Burnout syndrome and its relationship with the absence of quality of life, not working with \\ illness
Síndrome de burnout e sua relação com a ausência de qualidade de vida no trabalho de enfermagem

Ediléia de Jesus Sousa Barros ${ }^{1 *}$, Vera Lucia Rocha Silva Barbosa1, Elizana de Fátima Garcia Soares ${ }^{1}$.

\section{RESUMO}

Objetivo: Revisar e identificar a incidência de Síndrome de Burnout e a relação existente com o ambiente de trabalho do enfermeiro. Métodos: A pesquisa apresentada neste trabalho trata-se de uma revisão bibliográfica com características descritiva e exploratória de estudos publicados acerca da síndrome estudada no contexto da enfermagem. busca foi realizada por meio de literatura disponível em bases de dados na internet, sendo elas: Scientific Eletronic Library Online (SCIELO), Centro Latino-Americano e do Caribe de Informação em Ciência da Saúde (LILACS), e Bases de dados de Enfermagem (BDENF). Resultados: Através dos descritores, foram encontrados um total de 9.163 artigos nas bases de dados propostas. Após aplicação de critérios de inclusão e exclusão, foram excluídos 9.006 artigos, sendo 157 pré-selecionados, 58 repetidos e 19 selecionados. Considerações finais: Dentre todas as categorias profissionais existentes atualmente, os profissionais de enfermagem apresentam um alto índice de desenvolvimento da síndrome em questão. Portanto, diante dos resultados alcançados neste estudo é fundamental que haja um planejamento estratégico em prol dos profissionais de enfermagem vulneráveis a esta patologia, assim como, redução de carga horária, salário compatível, educação permanente atuante, comunicação adequada entre profissionais, família e empregador, além de melhoria nos recursos humanos e materiais.

Palavras-chave: Doenças profissionais, Burnout, Enfermagem do trabalho, Síndrome de esgotamento profissional.

\section{ABSTRACT}

Objective: To review and identify the incidence of Burnout Syndrome and its relationship with the nurse's work environment. Methods: The research presented in this work is a literature review with descriptive and exploratory characteristics of published studies about the syndrome studied in the context of nursing. The search was performed using literature available in internet databases, namely: Scientific Electronic Library Online (SCIELO), Latin American and Caribbean Center for Health Science Information (LILACS), and Nursing Databases (BDENF). Results: Through the descriptors, a total of 9,163 articles were found in the proposed databases. After applying the inclusion and exclusion criteria, 9,006 articles were excluded, being 157 preselected, 58 repeated and 19 selected. Final Considerations: Among all professional categories currently existing, nursing professionals have a high rate of development of the syndrome in question. Therefore, given the results achieved in this study, it is essential that there is a strategic planning in favor of nursing professionals who are vulnerable to this pathology, as well as a reduction in the workload, compatible salary, active permanent education, adequate communication between professionals, family and employer, in addition to improving human and material resources.

Keywords: Occupational diseases, Burnout, Nursing work, Burnout syndrome.

\section{RESUMEN}

Objetivo: Revisar e identificar la incidencia del Síndrome de Burnout y su relación con el entorno laboral del enfermero. Métodos: La investigación presentada en este trabajo es una revisión de la literatura con características descriptivas y exploratorias de estudios publicados sobre el síndrome estudiado en el contexto de la enfermería. La búsqueda se realizó utilizando la literatura disponible en bases de datos de Internet, a saber: Scientific Electronic Library Online (SCIELO), Centro Latinoamericano y del Caribe de Información en

\footnotetext{
${ }^{1}$ Faculdade FASIPE CPA, Cuiabá - MT. *E-mail: edileia.barros@hotmail.com
} 
Ciencias de la Salud (LILACS) y Bases de Datos de Enfermería (BDENF). Resultados: A través de los descriptores, se encontraron un total de 9.163 artículos en las bases de datos propuestas. Tras aplicar los criterios de inclusión y exclusión, se excluyeron 9.006 artículos, siendo 157 preseleccionados, 58 repetidos y 19 seleccionados. Consideraciones finales: Entre todas las categorías profesionales actualmente existentes, los profesionales de enfermería tienen una alta tasa de desarrollo del síndrome en cuestión. Por tanto, dados los resultados alcanzados en este estudio, es fundamental que exista una planificación estratégica a favor de los profesionales de enfermería vulnerables a esta patología, así como una reducción de la carga de trabajo, salario compatible, educación permanente activa, comunicación adecuada entre profesionales, familiares y empresarios, además de mejorar los recursos humanos y materiales.

Palabras clave: Enfermedades profesionales, Burnout, Trabajo de enfermería, Síndrome de burnout.

\section{INTRODUÇÃO}

A Síndrome do Esgotamento Profissional (SEP) ou como é mais conhecida Síndrome de Burnout (SB) vem sendo tema de investigação no cenário acadêmico por se tratar de um fator impactante na vida profissional do profissional da enfermagem. Essa síndrome surge do estresse decorrente do trabalho e pode ser reconhecida como uma psicopatologia ocupacional definida no grupo $\mathrm{V}$ da CID-10, sendo inclusa no Anexo II do artigo $2^{\circ}$ do Decreto 6.957/1999, regido pela Previdência Social, seu diagnóstico é pouco utilizado (MASLACH C, et al., 2012; MEDEIROS-COSTA ME, et al., 2017).

A SEP pode ser classificada como modelo teórico tridimensional, que se se ancora em três aspectos: 0 esgotamento emocional, a despersonalização e o não desempenho profissional. A exaustão emocional se apresenta como uma manifestação direta do estresse individual, sendo exteriorizadas sensações de estar além dos limites, com deterioração dos recursos físicos do indivíduo. Já a despersonalização está ligada em atitudes negativas que são direcionadas às pessoas destinatárias do trabalho, a despersonalização também se caracteriza como uma perda de compaixão para com os outros. Por fim, a realização profissional se relaciona a avaliações negativas do indivíduo quanto ao seu desempenho no trabalho e seu futuro naquela profissão. No entanto, essa classificação ainda não está bem definida na literatura (MASLACH C, et al., 2012; MEDEIROS-COSTA ME, et al., 2017).

O aumento da carga de trabalho entre os trabalhadores que está relacionado à distribuição das cargas horárias, precarização salarial e jornadas mensais maiores a que os demais, impõem um regime de trabalho desigual com maior desgaste físico e mental ao profissional. Paralelo a esses fatores ocorre também a necessidade de complementação da renda e a instabilidade empregatícia, fazendo com que estes profissionais tenham múltiplos empregos, gerando maior desgaste devido ao aumento da jornada de trabalho (ALVAREZ YDM, 2015; SOUZA M, et al., 2015).

Vale ressaltar que os profissionais que carregam tais desgastes físico e emocional, são corresponsáveis pelos seus comportamentos adotados, como fadiga, indiferença e raiva. Desgastes estes que advém de um desequilíbrio significativo das organizações, o que demonstra a fragilidade e vulnerabilidade no local de trabalho (OLIVEIRA LO e OLIVEIRA SMK, 2016).

A SB, segundo Medeiros-Costa ME, et al. (2017) é uma das principais psicopatologias no âmbito ocupacional, podendo advir de origens devido à exaustão emocional, à despersonalização, e à insatisfação pessoal, diante disto, foram criados diversos instrumentos para a avaliação e diagnóstico da SB, sendo o Maslach Burnout Inventory (MBI) o mais usado. Atualmente o MBI possui três versões, sendo: Human Services Survey (MBI_HSS) que contém 22 itens voltados para profissionais da área da saúde; Educators Survey (MBI-ES), dirigidas a profissionais da educação; e General Survey (MBI-GS), com apenas16 itens, sendo está uma pesquisa geral (GIL-MONTE PR, 2003).

Este estudo justifica-se pelo aumento do número de trabalhadores da equipe de enfermagem que convivem com tal Síndrome. Estatísticas da Previdência Social mostram a gravidade do problema quando afirmam que, nos anos de 2012 a 2016, 668.927 pessoas foram afastadas por distúrbios mentais e comportamentais, sendo 34.511 especificamente com SB, considerado, portanto, como a terceira causa de afastamento laboral (BRASIL, 2017). 
Se, de um lado é possível identificar fatores que desencadeiam a Síndrome de Burnout, e por outro, o aumento de tal patologia, um estudo sobre tal tema faz-se necessário. Diante disso, o objetivo deste estudo foi revisar e identificar a incidência de Síndrome de Burnout e a relação existente com o ambiente de trabalho do enfermeiro.

\section{MÉTODOS}

A pesquisa apresentada neste trabalho trata-se de uma revisão bibliográfica com características descritiva e exploratória de estudos publicados acerca da SB no contexto da enfermagem. Neste tipo de pesquisa, a partir de uma questão norteadora, o autor realiza uma busca de conhecimentos por meio da coleta de dados, durante a coleta o autor identifica, analisa e avalia o conteúdo e sua contribuição para a compreensão e explicação do problema investigado (KOCHE JC, 2011).

Assim, surge como questão: Qual a relação entre a Síndrome de Burnout e a ausência de qualidade de vida no trabalho de enfermagem? Diante disto, a revisão foi composta dos seguintes passos: realização da busca de artigos nas bases de dados; definição de critérios de inclusão e exclusão, posteriormente foi coletada informações extraídas dos artigos pré-selecionados e realizada a análise e interpretação dos dados encontrados.

A busca foi realizada por meio de literatura disponível em bases de dados na internet, através da Biblioteca Virtual em Saúde (Bireme). As bases utilizadas para a pesquisa foram: Scientific Eletronic Library Online (SCIELO), Centro Latino-Americano e do Caribe de Informação em Ciência da Saúde (LILACS), e Bases de dados de Enfermagem (BDENF). As buscas se deram no período de fevereiro a abril de 2020.

Para a busca de dados, utilizaram-se como descritores: doenças profissionais; Burnout; enfermagem do trabalho; síndrome de esgotamento profissional.

Os critérios de inclusão definidos para o estudo foram: artigos voltados para a questão norteadora que disponham dos descritores nos títulos e resumos dos artigos; ser publicado entre o período de 2015 a 2020; em um dos idiomas (português, inglês e espanhol) e estar disponibilizado na íntegra. Como critérios de exclusão foram descartadas teses e dissertações, além de artigos que destoaram da temática e objetivos do estudo. Artigos utilizados com período inferior a 2015 foram descritos devido sua grande relevância ao contexto proposto.

\section{RESULTADOS}

A soma total de estudos encontrados nas bases de dados chegou a 9.163 artigos. No entanto, após uma breve leitura dos títulos foi encontrado e excluído as duplicatas, em seguida, após uma leitura parcial outros artigos foram excluídos, já que não preenchiam os critérios deste estudo. Foram então selecionados 157 artigos que foram lidos minuciosamente, nesta etapa foram excluídos aqueles que fugiram da temática e não respeitaram o propósito deste estudo. Foram selecionados para compor a amostra da revisão os 19 artigos que preenchiam os critérios iniciais propostos e que foram lidos na íntegra (Quadro 1 e Quadro 2).

Quadro 1 - Total de artigos conforme palavra-chave e Base de dados.

\begin{tabular}{|c|c|c|c|c|c|}
\hline $\begin{array}{c}\text { Palavras-chaves/ } \\
\text { Base de dados }\end{array}$ & Burnout & $\begin{array}{c}\text { Doenças } \\
\text { profissionais }\end{array}$ & $\begin{array}{c}\text { Enfermagem } \\
\text { do trabalho }\end{array}$ & $\begin{array}{c}\text { Síndrome do } \\
\text { esgotamento } \\
\text { profissional }\end{array}$ & Total \\
\hline BDENF & 142 & 467 & 1.759 & 58 & $\mathbf{2 . 4 2 6}$ \\
\hline LILACS & 577 & 1.373 & 2.359 & 158 & $\mathbf{4 . 4 6 7}$ \\
\hline SCIELO & 531 & 416 & 1.262 & 61 & $\mathbf{2 . 2 7 0}$ \\
\hline Total & $\mathbf{1 . 2 5 0}$ & $\mathbf{2 . 2 5 6}$ & $\mathbf{5 . 3 8 0}$ & $\mathbf{2 7 7}$ & $\mathbf{9 . 1 6 3}$ \\
\hline
\end{tabular}

Fonte: Barros EJS, et al., 2021.

Quadro 2 - Filtro dos artigos encontrados.

\begin{tabular}{|c|c|c|c|c|}
\hline $\begin{array}{c}\text { Total de artigos } \\
\text { encontrados }\end{array}$ & Excluídos & Pré-selecionados & Repetidos & Selecionados \\
\hline 9.163 & 9.006 & 157 & 58 & 19 \\
\hline
\end{tabular}

Fonte: Barros EJS, et al., 2021. 
Dentre estes 19 artigos, 6 (31,57\%) foram encontrados na base de dados LILACS e SCIELO respectivamente, e 7 (36,84\%) na BDENF. Sendo 15 artigos realizados no Brasil (português), 3 no exterior (coreano, indonésio e espanhol) e 1 escrito por autores brasileiros e espanhóis. No que se refere ao ano de publicação, 6 artigos (31,57\%) foram publicados no ano de 2018 e 2019 respectivamente, 3 (15,78\%) no ano de 2017, 2 (10,52\%) no ano de 2015, e 1 artigo nos anos de 2016 e 2020 (5,26\% respectivamente) (Figura 1).

A metodologia mais encontrada foi a Revisão Integrativa com 8 artigos $(41,10 \%)$, seguida da quantitativa com 7 artigos (36,84\%), e transversal com 4 (21,05\%) (Figura 1).

Figura 1 - Fluxograma de busca e seleção dos artigos.

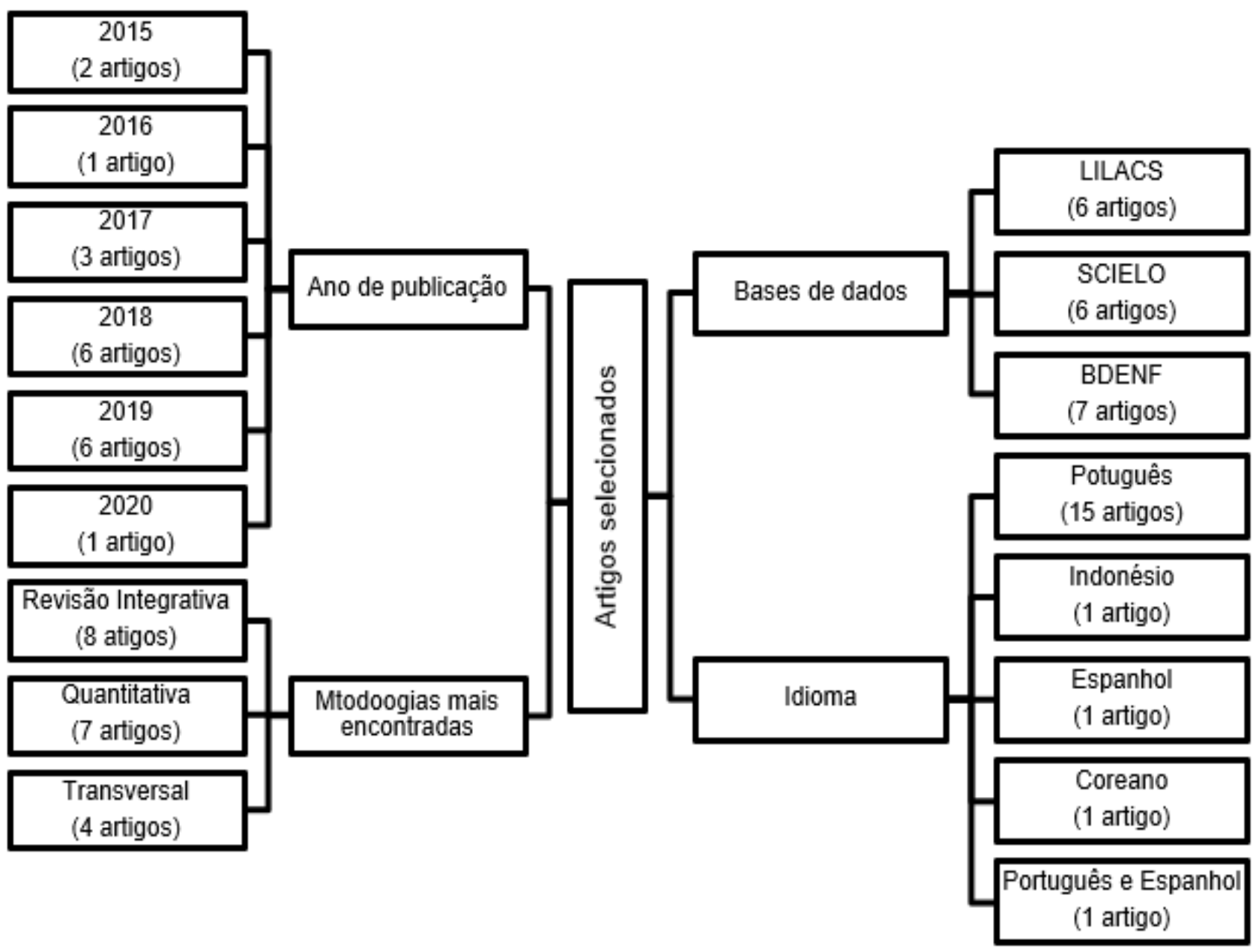

Fonte: Barros EJS, et al., 2021.

Dentre os 19 artigos selecionados, 15 artigos (78,94\%) tiveram como público-alvo profissionais da enfermagem, sendo 3 artigos (15,78\%) sobre o profissional enfermeiro, 12 artigos (63,15\%) sobre enfermeiros e técnicos de enfermagem. E 4 artigos (21,05\%) sobre a SB e a saúde do trabalhador no ambiente hospitalar.

Especificamente sobre o turno de trabalho destes profissionais, 8 artigos $(42,10 \%)$ fizeram esta análise, sendo que obtiveram o resultado de $100 \%$ dos entrevistados trabalhando no período diurno e/ou noturno.

Apenas 10 (52,63\%) artigos selecionados analisaram a presença dos sintomas característicos da SB, de modo que todos (100\%) os entrevistados apresentaram algum grau de exaustão emocional, despersonalização e não realização profissional.

Apresenta-se no Quadro 3, os artigos encontrados nas bases de dados descritas acima, colaborando para o desenvolvimento dos resultados e análise dos mesmos. 
Quadro 3 - Descrição dos artigos selecionados.

\begin{tabular}{|c|c|c|c|c|}
\hline Título & Autor (es) & $\begin{array}{l}\text { País/ Base de } \\
\text { dados }\end{array}$ & Tipo de estudo & Conclusão \\
\hline $\begin{array}{c}\text { Incidência da Síndrome de Burnout nos } \\
\text { profissionais de enfermagem: uma revisão } \\
\text { integrativa }\end{array}$ & $\begin{array}{l}\text { Oliveira RF, et al., } \\
\text { (2017). }\end{array}$ & Brasil / LILACS & $\begin{array}{l}\text { Revisão integrativa da } \\
\text { literatura }\end{array}$ & $\begin{array}{l}\text { É necessário fazer uma reflexão a fim de buscarmos, enquanto } \\
\text { pesquisadores, acadêmicos e profissionais de enfermagem, o que pode ser } \\
\text { feito para amenizar a incidência dessa doença que tem aumentado a cada dia } \\
\text { mais nesses trabalhadores. }\end{array}$ \\
\hline $\begin{array}{l}\text { Burnout entre os profissionais de } \\
\text { enfermagem em hospitais no Brasil }\end{array}$ & $\begin{array}{l}\text { Dutra HC, et al. } \\
\text { (2019). }\end{array}$ & Brasil / LILACS & Estudo transversal & $\begin{array}{l}\text { Características pessoais e profisssionais foram relacionadas ao Burnout entre } \\
\text { profissionais de enfermagem. É importante desenvolver ações capazes de } \\
\text { minimizar a ocorrência de Burnout entre os profissionais de enfermagem. }\end{array}$ \\
\hline $\begin{array}{l}\text { Síndrome de Burnout no pessoal de } \\
\text { enfermagem e sua relação com quatro } \\
\text { variáveis laborais }\end{array}$ & $\begin{array}{l}\text { Rivas E e Barraza- } \\
\text { Macias A (2018). }\end{array}$ & $\begin{array}{l}\text { México / } \\
\text { LILACS }\end{array}$ & $\begin{array}{l}\text { Correlacional, } \\
\text { transversal e não } \\
\text { experimental }\end{array}$ & $\begin{array}{l}\text { As condições laborais podem desencadear situações estressantes que a } \\
\text { longo prazo podem gerar a SB, que é o caso de variáveis como o turno em } \\
\text { que se trabalha ou o tipo de função laboral que exerce. A aparição desta } \\
\text { Síndrome afeta o desempenho profissional do profissional de enfermagem. }\end{array}$ \\
\hline $\begin{array}{l}\text { A relação de Síndrome de Burnout com os } \\
\text { profissionais de enfermagem: revisão } \\
\text { integrativa }\end{array}$ & $\begin{array}{l}\text { Larré MC, et al. } \\
\qquad(2018) \text {. }\end{array}$ & Brasil / LILACS & Revisão integrativa & $\begin{array}{l}\text { Os profissionais de enfermagem precisam buscar informações e } \\
\text { conhecimentos acerca da doença afim de promover a prevenção, assim como } \\
\text { os gestores precisam lançar metas eficazes, incluindo os métodos de redução } \\
\text { da carga horária, a melhoria nos recursos humanos e materiais, o plano de } \\
\text { salário compatível, além da educação permanente. }\end{array}$ \\
\hline $\begin{array}{l}\text { Prevalência do estresse e Síndrome de } \\
\text { Burnout em enfermeiros no trabalho } \\
\text { hospitalar em turnos }\end{array}$ & $\begin{array}{l}\text { Bezerra CMB, et al. } \\
\text { (2019). }\end{array}$ & Brasil / LILACS & Descritivo e analítico & $\begin{array}{l}\text { O nível de estresse entre os enfermeiros e as três dimensões da síndrome } \\
\text { foram avaliados como nível médio nos turnos diurno e noturno. Houve } \\
\text { correlação estatisticamente significativa entre estresse e Burnout. }\end{array}$ \\
\hline Síndrome de Burnout & $\begin{array}{l}\text { Pêgo FPL e Pêgo DR } \\
\text { (2016). }\end{array}$ & Brasil / LILACS & Revisão bibliográfica & $\begin{array}{l}\text { A síndrome necessita ser reconhecida como doença, para que possam ser } \\
\text { implementadas medidas para sua prevenção. }\end{array}$ \\
\hline $\begin{array}{l}\text { Predisposição para Síndrome de Burnout } \\
\text { na equipe de enfermagem do Serviço de } \\
\text { Atendimento Móvel de Urgência }\end{array}$ & $\begin{array}{l}\text { Silva FG, et al. } \\
\text { (2019). }\end{array}$ & Brasil / BDENF & Exploratória descritiva. & $\begin{array}{l}\text { A equipe de enfermagem do SAMU tem predisposição a SB, sendo primordial } \\
\text { o enfrentamento dos fatores estressores da Enfermagem e adoção de } \\
\text { medidas preventivas para reduzir o estresse laboral. }\end{array}$ \\
\hline $\begin{array}{l}\text { Estresse e Síndrome de Burnout em } \\
\text { profissionais de enfermagem que atuam na } \\
\text { nefrologia: uma revisão integrativa }\end{array}$ & $\begin{array}{l}\text { Ruback SP, et al. } \\
(2018)\end{array}$ & Brasil / BDENF & Revisão integrativa & $\begin{array}{l}\text { Com este resultado, espera-se ampliar a visão sobre o estresse e Burnout em } \\
\text { enfermeiros que atuam em nefrologia identificando os fatores que podem } \\
\text { influenciar na assistência. }\end{array}$ \\
\hline $\begin{array}{l}\text { Síndrome de Burnout: uma análise } \\
\text { reflexiva }\end{array}$ & $\begin{array}{l}\text { Menezes PCM, et al. } \\
\text { (2017). }\end{array}$ & Brasil / BDENF & $\begin{array}{l}\text { Qualitativo, descritivo, } \\
\text { tipo análise reflexiva } \\
\text { narrativa }\end{array}$ & $\begin{array}{l}\text { Verifica-se alta associação entre Burnout e trabalho assistencial na saúde por } \\
\text { profissionais que exercem papéis de alta complexidade/responsabilidade } \\
\text { lidando com pacientes dos mais variados problemas de saúde, tanto no } \\
\text { âmbito da emergência quanto de setores de alto risco de doenças infecto- } \\
\text { contagiosas ou até mesmo em UTI. À somatória de plantões noturnos } \\
\text { estressantes, contato diário com enfermos, sofrimento, possibilidade da morte } \\
\text { os deixa em condição propícia para o desenvolvimento da SB. }\end{array}$ \\
\hline $\begin{array}{l}\text { Fatores desencadeantes da Síndrome de } \\
\text { Burnout em enfermeiros }\end{array}$ & $\begin{array}{l}\text { Paiva JDM, et al. } \\
\text { (2019). }\end{array}$ & Brasil / BDENF & $\begin{array}{l}\text { Bibliográfico, descritivo, } \\
\text { revisão integrativa }\end{array}$ & $\begin{array}{l}\text { Conclui-se a necessidade de os próprios profissionais de Enfermagem se } \\
\text { conscientizarem sobre a importância de cuidar da sua saúde no ambiente de } \\
\text { trabalho, minimizando o risco de desenvolvimento da SB. }\end{array}$ \\
\hline
\end{tabular}




\begin{tabular}{|c|c|c|c|c|}
\hline Título & Autor (es) & $\begin{array}{l}\text { País/ Base de } \\
\text { dados }\end{array}$ & Tipo de estudo & Conclusão \\
\hline $\begin{array}{c}\text { Síndrome de Burnout em profissionais de } \\
\text { enfermagem }\end{array}$ & $\begin{array}{l}\text { Costa SMS, et al. } \\
\qquad(2020)\end{array}$ & Brasil / BDENF & $\begin{array}{l}\text { Quantitativo, descritivo, } \\
\text { transversal }\end{array}$ & $\begin{array}{l}\text { Conclui-se que houve predominância do nível moderado nas três dimensões } \\
\text { do Burnout, resultado que se mostra preocupante, uma vez que, como } \\
\text { defendido por Betty Neuman em sua teoria, a estabilidade do sistema e a } \\
\text { necessidade deste ser mantido em equilíbrio são essenciais para evitar o } \\
\text { adoecimento psíquico, inclusive, no ambiente laboral. }\end{array}$ \\
\hline $\begin{array}{l}\text { Saúde do trabalhador no ambiente } \\
\text { hospitalar: fatores de risco para Síndrome } \\
\text { de Burnout }\end{array}$ & $\begin{array}{l}\text { Santos EM, et al. } \\
(2018) .\end{array}$ & Brasil / BDENF & $\begin{array}{l}\text { Analítico, } \\
\text { observacional, } \\
\text { transversal e } \\
\text { quantitativo }\end{array}$ & $\begin{array}{l}\text { O desafio da promoção da saúde no trabalho e da prevenção de Burnout } \\
\text { torna-se ainda maior na medida em que é exigido um diálogo permanente } \\
\text { entre o planejamento, a execução e gestão. }\end{array}$ \\
\hline $\begin{array}{l}\text { Síndrome de Burnout e o trabalho em } \\
\text { turnos na equipe de enfermagem }\end{array}$ & $\begin{array}{l}\text { Vidotti V, et al. } \\
\qquad(2018)\end{array}$ & Brasil / BDENF & Transversal & $\begin{array}{l}\text { Os fatores psicossociais e do contexto laboral, sobretudo o baixo apoio } \\
\text { social, tiveram associação com as dimensões da síndrome entre os } \\
\text { profissionais de enfermagem de ambos os turnos. }\end{array}$ \\
\hline $\begin{array}{l}\text { Estresse ocupacional em profissionais de } \\
\text { enfermagem }\end{array}$ & $\begin{array}{l}\text { Lappa-Rodriguez EO, } \\
\text { et al. (2018). }\end{array}$ & Brasil / SCIELO & $\begin{array}{l}\text { Descritiva e } \\
\text { correlacional }\end{array}$ & $\begin{array}{l}\text { A enfermagem se revelou como uma profissão de risco para o estresse ocupa- } \\
\text { cional, sofrendo diversas influências dos fatores analisados, relacionados à } \\
\text { organização e ao processo de trabalho. }\end{array}$ \\
\hline $\begin{array}{l}\text { Síndrome de Burnout em trabalhadores de } \\
\text { enfermagem brasileiros e espanhóis }\end{array}$ & $\begin{array}{l}\text { Mosteiro-Baldonedo } \\
\text { M, et al. (2019). }\end{array}$ & $\begin{array}{l}\text { Brasil e } \\
\text { Espanha / } \\
\text { SCIELO }\end{array}$ & $\begin{array}{l}\text { Quantitativo, } \\
\text { transversal e } \\
\text { comparativo }\end{array}$ & $\begin{array}{l}\text { Apesar dos trabalhadores de enfermagem brasileiros e espanhóis pontuarem } \\
\text { níveis baixos de Despersonalização e elevados de Realização Profissional, } \\
\text { verifica-se níveis médios de Exaustão Emocional, indicando um fator } \\
\text { preventivo importante a ser trabalhado, uma vez que a Exaustão Emocional é } \\
\text { considerada o primeiro estágio do Burnout. }\end{array}$ \\
\hline $\begin{array}{l}\text { Trabalho em turnos de profissionais de } \\
\text { enfermagem e a pressão arterial, Burnout } \\
\text { e transtornos mentais comuns }\end{array}$ & $\begin{array}{l}\text { Nascimento JOV, et } \\
\quad \text { al. (2019). }\end{array}$ & Brasil / SCIELO & Transversal & $\begin{array}{l}\text { O trabalho por turnos foi associado a uma maior prevalência de fatores } \\
\text { negativos relacionados ao trabalho, hábitos e estilos de vida inadequados e } \\
\text { alterações no sangue do sono pressão. }\end{array}$ \\
\hline $\begin{array}{l}\text { Estresse ocupacional entre auxiliares e } \\
\text { técnicos de enfermagem: enfrentamento } \\
\text { focado no problema }\end{array}$ & $\begin{array}{l}\text { Teixeira CAB, et al. } \\
(2015) .\end{array}$ & Brasil / SCIELO & $\begin{array}{l}\text { Quantitativo e } \\
\text { correlacional }\end{array}$ & $\begin{array}{l}\text { Neste estudo, a maior escolaridade e o número de filhos se associaram com } \\
\text { um alto uso de estratégias de enfrentamento focadas no problema, mostrando } \\
\text { uma menor vulnerabilidade os fatores de estresse ao trabalho. }\end{array}$ \\
\hline $\begin{array}{l}\text { Relação entre profissionais estresse e } \\
\text { humor depressivo entre estagiários e } \\
\text { residentes em um hospital terciário, Coréia } \\
\text { do Sul }\end{array}$ & Kim K, et al. (2015). & $\begin{array}{l}\text { Coréia / } \\
\text { SCIELO }\end{array}$ & Transversal & $\begin{array}{l}\text { Os residentes do hospital experimentam um alto grau de estresse } \\
\text { ocupacional, levando a um humor deprimido devido a vários fatores de risco. } \\
\text { Portanto, é essencial reconhecer o estresse ocupacional dos residentes } \\
\text { desde o início, incentivar a competição positiva e o apoio social e de pares, } \\
\text { além de ajudar a melhorar a capacidade dos residentes de lidar com o } \\
\text { estresse. }\end{array}$ \\
\hline $\begin{array}{l}\text { O efeito da ambiguidade do papel para o } \\
\text { desempenho dos enfermeiros através de } \\
\text { compromisso organizacional: um estudo } \\
\text { sobre hospital geral do Dr Saiful Anwar, } \\
\text { Malang, Indonésia }\end{array}$ & $\begin{array}{l}\text { Fauzan A, et al. } \\
\qquad(2017)\end{array}$ & $\begin{array}{l}\text { Indonésia / } \\
\text { SCIELO }\end{array}$ & Quantitativo, analítico & $\begin{array}{l}\text { Os resultados deste estudo são os seguintes: (1) a ambiguidade do papel tem } \\
\text { um efeito significativo sobre o comprometimento; e (2) a ambiguidade do } \\
\text { papel tem um efeito significativo no desempenho dos enfermeiros. }\end{array}$ \\
\hline
\end{tabular}

Fonte: Barros EJS, et al., 2021. 


\section{DISCUSSÃO}

\section{O conceito da síndrome de burnout}

A SB é um distúrbio psíquico de cunho depressivo caracterizado pelo adoecimento do profissional, gerando assim repercussões negativas que podem ser entendidas no déficit da qualidade da assistência prestada à população, além de sentimentos de agressividade e desprezo com a equipe, prejudicando a vida pessoal, social e ocupacional do indivíduo. Esta síndrome é ocasionada por fatores biopsicossociais associados ao ambiente de trabalho, e de acordo com esses autores, somente o perfil individual não é desencadeador de tal patologia, o que a torna um problema de saúde pública e intimamente ligada a gestão organizacional dos serviços de trabalho (SILVA FG, et al., 2019).

Oliveira RF, et al. (2017), descreve a exaustão emocional como o esgotamento dos sentimentos vividos no cotidiano. Estes mesmos estudiosos afirmam que acompanhada de múltiplos sintomas, a SB afeta o profissional em seus âmbitos físicos e/ou psíquicos, gerando uma queda do seu rendimento e capacidade de produção. Esses sintomas de SB podem ser: dor no peito, pressão arterial alta, perda ou excesso de apetite, dificuldade com o sono (SANTOS EM, et al., 2018).

Os sintomas da SB são considerados uma resposta ao estresse laboral, surgindo quando as estratégias funcionais de enfrentamento para lidar com solicitações excessivas falham, levando ao esgotamento dos sentimentos de emoção para lidar com as situações do dia a dia. Esses sintomas podem afetar o indivíduo de forma psíquica, física ou das duas maneiras. A partir daí há uma perda da capacidade de produção e vigor no trabalho (PÊGO FPL e PÊGO DR, 2016).

Segundo Oliveira RF, et al. (2017), o indivíduo envolve bloqueio e sentimentos negativos do trabalhador consigo mesmo e com os outros. Como forma de autoproteção, esse mesmo indivíduo passa a isolar-se dos outros e começa a manter uma atitude fria e com conduta egoísta, tratando os demais de maneira ríspida e desumanizada.

\section{$O$ conceito e etiologia do estresse}

Segundo Ruback SP, et al. (2018), em 1934 o cientista endocrinologista Hans Selye introduz a palavra estresse na área da saúde. A existência do estresse surgiu através do interesse de estudar as queixas e sintomas apresentados por alguns de seus pacientes. Ruback SP, et al. (2018) também relatam que, depois de Selye diversas teorias sobre o estresse foram desenvolvidas, entre essas teorias está a de Lazarus (1966) que dividiu em três organizações: as que consideram o estresse uma condição fisiológica do organismo; as que consideram que o estresse resulta da ação do ambiente; e as teorias interacionistas, como a teoria transacional de Lazarus que diz que o estresse é produto da diferença entre a relação das demandas e o controle interno que o indivíduo tem, são as teorias mais difundidas na atualidade.

A teoria transacional também é conhecida como a teoria do coping ou enfrentamento, quando as estratégias de enfrentamento do repertório de uma pessoa são adotadas e não são suficientes para a resolução de um problema, o estresse se desenvolve, quando as estratégias funcionam a adversidade é considerada um desafio e pode ser também um estímulo (RUBACK SP, et al., 2018).

Para Pêgo FPL e Pêgo DR (2016), é importante frisar que o trabalho deveria ser percebido positivamente como um ato prazeroso, porém, para muitos, devido diversas circunstancias como o clima organizacional e emocional no ambiente de trabalho, o trabalho desperta sentimentos negativos e sensação de sacrifício, levando ao adoecimento. De acordo com Kim K, et al. (2015), entre os processos de interação entre o trabalhador e seu ambiente de trabalho decorre o estresse ocupacional, que segundo Teixeira CAB, et al. (2015) é o estado reacional biológico deletério e caracterizado como um agravamento multifatorial decorrente de processos interacionais entre o trabalhador e seu ambiente (KIM, K, et al., 2015).

\section{O estresse ocupacional e a síndrome de burnout na enfermagem}

Neste cenário, de acordo com Lappa-Rodriguez EO, et al. (2018), o estresse ocupacional tornou-se um problema real na enfermagem, por ser uma profissão que exige do profissional trabalho exaustivo nas mais diversas atribuições desenvolvidas. Por ser a SB uma reação psicológica bem conhecida entre profissionais 
da saúde, segundo Menezes PCM, et al. (2017), são comuns fatores desencadeantes como, ambiente e interação, encerramento da relação enfermagem-paciente, falta de coerência, lento desenvolvimento profissional, falta de autonomia e carga de trabalho excessiva.

Portanto, Fauzan A, et al. (2017) e Lappa-Rodriguez EO, et al. (2018) concordam que, o estresse no trabalho do profissional de enfermagem ocorre constantemente em toda as áreas e categorias, devido o ambiente e as condições de trabalho, causando problemas físico e mental nesses profissionais.

Consequentemente, segundo Oliveira RF, et al. (2017), pode-se afirmar que, a SB afeta negativamente tanto na assistência ao paciente quanto na relação com os colegas, interferindo também na qualidade de vida e saúde do trabalhador em todas as áreas de sua vida, pois gera sensação de cansaço e esgotamento físico e psicológico, diante disto, a SB torna-se um importante problema de saúde pública.

Desta maneira, vale ressaltar que de acordo com Ruback SP, et al. (2018), a SB e o estresse podem predispor enfermeiros a piores condições de saúde, favorecendo ao ciclo vicioso, o que pode levar à máqualidade de assistência ao paciente e aumento dos gastos organizacionais. Ainda conforme Ruback SP, et al. (2018), os transtornos mentais e comportamentais se despontam como uma das causas principais de afastamento entre os trabalhadores, sendo mais frequente entre trabalhadores que atendem ao público, especialmente entre aqueles que prestam cuidados diretos aos pacientes.

A enfermagem é uma categoria profissional que exerce suas atividades em ritmo acelerado, e de acordo com Oliveira RF, et al. (2017), na maioria das vezes sobrecarregada devido a altas demandas nos serviços de saúde e que lida diariamente em seu âmbito de trabalho com diversos fatores estressores, como a dor, o sofrimento, a morte, a exposição a pressões por parte dos pacientes e seus respectivos familiares, dificuldade no contato e relacionamento com a chefia e até mesmo com os próprios colegas de trabalho, esse último em especial quando se trata da supervisão e coordenação de enfermagem, uma vez que o enfermeiro é a referência técnica para todo o restante da equipe.

Sobre o turno de trabalho, ao se questionar as variáveis laborais, relacionadas à SB, Rivas E e BarrazaMacias A (2018) dizem que, os principais fatores apontados como causas de grande influência para tal desenvolvimento foi o turno de trabalho e a situação laboral.

Tal categoria possui jornadas de trabalho na maioria das vezes exaustiva, devido ao grande volume de pacientes e o pouco tempo de descanso (RUBACK SP, et al., 2018). Além disso, Menezes PCM, et al. (2017) afirmam que, mudança de turnos causa uma série de mudanças na vida do profissional, afetando sua saúde e qualidade de vida, pois altera o ritmo e a qualidade do sono, gerando no profissional estresse e insatisfação.

Para Dutra HC, et al. (2019), os profissionais que trabalham no período noturno o fazem, muitas vezes, a fim de conciliar com outro vínculo empregatício, bem como com convivências familiares. Porém, este mesmo autor defende que o trabalho realizado em neste turno pode acarretar uma série de mudanças fisiológicas, prejudicando assim a sensação de bem-estar e mental destes trabalhadores.

Os profissionais que trabalhavam durante a noite evidenciam altos índices da SB em comparação com os trabalhadores diurnos, e de acordo com Larré MC, et al. (2018), a fadiga e problemas de saúde são desencadeados pela queda do tempo de sono e sua qualidade, o que explica os altos índices de SB em trabalhadores noturnos.

Porém, no estudo de Vidotti V, et al. (2018), muitos profissionais de enfermagem concordam que trabalhar no turno noturno não é um ponto negativo, pois o horário permite a conciliação entre seus horários e sua vida tanto profissional quanto pessoal. Além disso, o horário garante melhor salário, menor supervisão e mais aproximação e interação com a equipe, devido à baixa demanda. Os autores também apontam níveis mais elevados entre os trabalhadores de enfermagem do período diurno, tendo como fatores causadores: alta demanda, baixa qualidade no sono e a pouca autonomia no cotidiano laboral, ocasionando um aumento na exaustão emocional.

Os dados acima corroboram com outra pesquisa onde os profissionais do diurno apresentaram maior índice de exaustão e despersonalização quando comparados com os do noturno, já a questão de realização profissional e estresse, o resultado foi mediano para os dois turnos (BEZERRA CMB, et al., 2019). 
Tais dados vão de encontro a uma outra pesquisa onde a maioria $(79,2 \%)$ apresentou nível moderado de despersonalização. Deve-se considerar, entretanto, que $22,7 \%$ dos profissionais estudados apresentaram baixa realização profissional e, consequentemente, podem estar inseguros na realização de seu trabalho, como também podem lidar de forma negativa com situações de conflito e podem sentir-se sobrecarregados (COSTA SMS, et al., 2020).

O prevalecimento do tabagismo, sono inadequado devido alterações, a necessidade de alerta e alimentação fora de hora e inadequada, de acordo com Nascimento JOV, et al. (2019) são fatores apresentados por profissionais que trabalham em turnos, e provavelmente é concernente ao trabalho pelas adaptações em que o indivíduo passa para que possa apresentar desempenho qualificado nas atividades laborais, gerando consequências negativas na qualidade de vida desse sujeito.

Compreende-se que o bem-estar físico e emocional no ambiente de trabalho não depende somente de um fator, ou seja, diz respeito a uma relação simultânea entre o profissional e a instituição, constituindo-se um desafio entre as partes envolvidas (PAIVA JDM, et al., 2019).

De acordo com Oliveira RF, et al. (2017), a ausência de autonomia, a sobrecarga de trabalho e a má definição do papel desses profissionais, além do tempo minimizado para a realização das ações assistenciais e alguns determinantes como a elevada demanda de pacientes, a limitação de recursos humanos, podem comprometer a qualidade do serviço prestado, levam à exaustão física e emocional (LAPA-RODRIGUEZ et al., 2018), e fazem com que esta seja uma das profissões com os maiores índices da SB.

Outro fator importante que pode contribuir para situações de estresse no profissional de enfermagem é o trabalho em equipe, pois interdependência nas atividades realizadas pelos profissionais que formam a equipe pode gerar estresse nos colegas, pois essa interdependência pode interferir no resultado esperado, porém, para muitos, a interdependência pode levar a realização pessoal (DUTRA HC, et al., 2019).

Menezes PCM, et al. (2017) mencionam ainda que, o profissional de saúde almeja por resolução de problemas durante o seu plantão, sob condições de estresse e em um curto espaço de tempo, exercendo ainda múltiplas funções.

Silva FG, et al. (2019), também corroboram com a temática ao afirmarem que a falta de realização profissional em altos níveis pode gerar sensação de incapacidade, baixa autoestima, desmotivação e infelicidade no trabalho, afetando a habilidade e a destreza quando associado a sentimentos de insensibilidade em relação aos clientes ou colegas de trabalho e frustação podem ser desencadeadores dessa síndrome.

Oliveira RF, et al. (2017) relatam que, podem ser destacados como outros fatores contribuintes para o tal desencadeamento da Síndrome, a estrutura do local de trabalho, a indisponibilidade de materiais para o cuidado de qualidade, duplas e longas jornadas de trabalho, sobrecarga de trabalho, salários inadequados, falta de estrutura física e material podem vir a ocasionar o adoecimento mental desses trabalhadores e consequentemente a SB. Portanto é indispensável um bom planejamento da escala dos profissionais, bem como dimensionamento pessoal (PAIVA JDM, et al., 2019).

A respeito Lappa-Rodriguez EO, et al. (2018) dizem que, os recursos humanos desempenham papel fundamental nos serviços de saúde e sua inadequada distribuição limitam sobremaneira o desempenho do profissional justificando sua significância e influência nos fatores de estresse analisados. Porém, estes mesmos autores, supõe-se que estando o profissional satisfeito com seu ambiente de trabalho, nas relações com os colegas, entre outras variáveis de satisfação, há probabilidade de que estas situações positivas transformem as pressões em fonte de crescimento emocional e profissional.

Portanto, para os autores Paiva JDM, et al. (2019), cria-se, desta forma, a necessidade de promover ações corretivas para lidar com o aumento de estresse no trabalho, a fim de minimizar as potenciais consequências psicológicas e preservar a satisfação do trabalho realizado, caracterizado na Enfermagem pela arte do cuidar.

Oliveira RF, et al. (2017) afirmam que, os profissionais de enfermagem lutam por reconhecimento, valorização e consequentemente aumento do salário, porém as dificuldades encontradas comprometem e desmotivam tal luta. Perante tantos desafios, os profissionais da enfermagem estão entre os mais atingidos pela SB, tendo como principal causa a sobrecarga, a falta de autonomia e a má gestão. 
E segundo Mosteiro-Baldonedo M, et al. (2019), dentro da enfermagem, a categoria com maior incidência da síndrome é a de técnico em enfermagem, pois desenvolvem atividades de assistência direta ao paciente, como técnicas de limpeza e higiene, causando maior desgaste físico. Além disso, a pressão direta durante a supervisão rigorosa e a qualidade do atendimento, tanto pelos usuários quanto pelo supervisor, também é um elemento capaz de aumentar o stress nesses trabalhadores.

\section{CONSIDERAÇÕES FINAIS}

Dentre todas as categorias profissionais existentes atualmente, os profissionais de enfermagem apresentam um alto índice de desenvolvimento da Síndrome estudada. Por ser uma psicopatologia no âmbito ocupacional advinda de exaustação emocional, despersonalização e insatisfação pessoal, somente o perfil individual não é desencadeador de tal patologia, sendo está intimamente ligada ao ambiente de trabalho. Os principais fatores apontados como causa de grande influência para tal desenvolvimento foram turno, equipe e ambiente de trabalho. Tendo uma maior incidência na categoria técnico de enfermagem. É de fundamental importância que aconteça um incentivo de novos estudos sobre esta temática, aprofundando em subtemas como carga horária excessiva, contato permanente com o sofrimento alheio, baixa remuneração, insatisfação profissional e o absenteísmo do mesmo.

\section{REFERÊNCIAS}

1. ALVAREZ YDM. Enfermeria en America Latina: una mirada al horizonte. Rev. Enferm., 2015; 33(2): 295-305.

2. BEZERRA CMB, et al. Prevalência do estresse e Síndrome de Burnout em enfermeiros no trabalho hospitalar em turnos. Rev. Min. Enferm., 2019; 23: 1232.

3. BRASIL. Ministério da Fazenda. Adoecimento mental e trabalho: a concessão de benefícios por incapacidade relacionados a transtornos mentais e comportamentais entre 2012 e $2016 / 1^{\circ}$ Boletim quadrimestral sobre benefícios por incapacidade. 2017. Disponível em: http://www.previdencia.gov.br/wpcontent/uploads/2017/04/1\%C 2\%BA-boletim-quadrimestral.pdf. Acesso em 02 de agosto de 2021.

4. COSTA SMS, et al. Síndrome de Burnout em profissionais de enfermagem. Rev. Enferm. UFPE onlline, 2020;14:243145.

5. DUTRA HC, et al. Burnout entre profissionais de enfermagem em hospitais no Brasil. Rev. Cuid., 2019; $10(1): 585$.

6. FAUZAN A, et al. The effect of role ambiguity towards the performance of nurses through organizational commitment: a study on regional general hospitalof dr. saiful anwar, malang, indonesia. Russian Journal of Agricultural and Socio-Economic Sciences (RJOAS), 2017; 6(66); 234-8.

7. GIL-MONTE PR. El Síndrome de Quemarse por el trabajo em enfermería. Rev. Elet. Inter. Ação Psy, $2003 ; 1$ (1): 19-33.

8. KIM, K, et al. Relationship between occupational stress and depressive mood among interns and residents in a tertiary hospital, Seoul, Korea. Clin. Exp. Emerg. Med., 2015; 2:117-22.

9. KOCHE JC. Fundamentos de metodologia científica: teoria da ciência e iniciação científica. Petrópolis, RJ: Vozes, 2011.

10. LAPPA-RODRIGUEZ EO, et al. Estresse ocupacional em profissionais de enfermagem. Rev. Enferm. UERJ, $2018 ; 26: 19404$.

11. LARRÉ MC, et al. A relação da Síndrome de Burnout com os profissionais de enfermagem: revisão integrativa. Revista Nursing, 2018; 21(237): 2018-2023.

12. MASLACH C, et al. Making a significant difference with burnout interventions: researcher and practitioner collaboration. J. Organ. Behav., 2012; 33(2): 296-300.

13. MEDEIROS-COSTA ME, et al. Occupational Burnout Syndrome in the nursing context: an integrative literature review. Rev. da Esc. Enferm. da USP, 2017; 51: 1-12.

14. MENEZES PCM, et al. Síndrome de Burnout: uma análise reflexiva. Rev. Enferm. UFPE online, 2017;11(12): 5092-101.

15. MOSTEIRO-BALDONEDO M, et al. Síndrome de Burnout entre trabalhadores de enfermagem brasileiros e espanhóis. Rev. Latino-Am. Enfermagem, 2019; 27: 3192.

16. NASCIMENTO JOV, et al. Turnos de trabalho dos profissionais de enfermagem e hipertensão, burnout e transtornos mentais comuns. Ver. Esc. Enferm. USP, 2019; 53: 03443.

17. OLIVEIRA LO, OLIVEIRA SMK. A Síndrome de Burnout nas Organizações. Rev. Maieutica, 2016; 4(1): 17- 26.

18. OLIVEIRA RF, et al. Incidência da Síndrome de Burnout nos profissionais de enfermagem: uma revisão integrativa. Revista de Enfermagem do Centro-Oeste Mineiro, 2017; 7: 1383.

19. PAIVA JDM, et al. Fatores desencadeantes da Síndrome de Burbout em enfermeiros. Rev. Enferm. UFPE online, 2019; 13(1): 483-90.

20. PÊGO FPL, PÊGO DR. Síndrome de Burnout. Rev. Bras. Med. Trab., 2016; 14(2): 171-6.

21. RIVAS E, BARRAZA-MACIAS A. Síndrome de burnout em trabalhadores de enfermagem e sua relação com quatro variáveis laborais. Doente univ., 2018; 15(2): 136-146.

22. RUBACK SP, et al. Estresse e Síndrome de Burnout em profissionais de enfermagem que atuam na nefrologia: uma revisão integrativa. Rev. Pesqui.: Cuid. Fundamm., 2018; 10(3): 889-899.

23. SANTOS EM, et al. Saúde do trabalhador no ambiente hospitalar: fatores de risco para síndrome de burnout. Nursing, 2019; 2572-2576.

24. SILVA FG, et al. Predisposição para síndrome de Burnout na equipe de enfermagem do serviço de atendimento móvel de urgência. Enferm. Foco, 2019; 10 (1): 40-45.

25. SOUZA M, et al. Suffering and precariousness at work in nursing. Revista De Pesquisa: Cuidado e Fundamental, 2015; 7(1): 2072-2082.

26. TEIXEIRA CAB, et al. Occupational stress among nursing technicians and assistants: coping focused on the problem. Invest. Educ. Enferm., 2015; 33:28-34.

27. VIDOTTI V, et al. Síndrome de Burnout e o trabalho em turnos na equipe de enfermagem. Rev. Latino-Am. Enfermagem, 2018; 26: 3022. 